\title{
Effects of recombinant bovine interleukin-8 (rblL-8) treatment on health, metabolism, and lactation performance in Holstein cattle III: Administration of rblL-8 induces insulin resistance in bull calves
}

\author{
M. Zinicola, ${ }^{1}$ P. R. Menta, ${ }^{1}$ B. L. Ribeiro, ${ }^{1}$ Y. Boisclair, ${ }^{2}$ and R. C. Bicalho ${ }^{1 *}$ \\ ${ }^{1}$ Department of Population Medicine and Diagnostic Sciences, Cornell University, Ithaca, NY 14853-6401 \\ ${ }^{2}$ Department of Animal Science, Cornell University, Ithaca, NY 14853-6401
}

\section{ABSTRACT}

Our previous work has suggested that recombinant bovine interleukin-8 (rbIL-8) treatment might influence cow metabolism. Therefore, this study was conducted to initially assess the effects of systemic administration of rbIL- 8 on response to a glucose challenge, blood metabolites, insulin, growth hormone, insulin-like growth factor-1, immune cell populations, and inflammatory parameters in Holstein bull calves. Calves from $30 \pm$ $6 \mathrm{~d}$ of life were individually housed and randomly allocated to 1 of 2 treatment groups: rbIL- 8 (rbIL-8, n $=10$ ) and control (CTR, $\mathrm{n}=8)$. Calves assigned to the rbIL- 8 group received 1 s.c. injection $(\mathrm{d} 1,0900 \mathrm{~h})$ and 6 i.v. injections (d 1 at $1600 \mathrm{~h}, \mathrm{~d} 2$ and 3 at 0900 $\mathrm{h}$ and $1600 \mathrm{~h}$, and $\mathrm{d} 4$ at $0900 \mathrm{~h})$ of rbIL-8 $(4 \mu \mathrm{g} / \mathrm{kg}$ of body weight), whereas the CTR group received $2 \mathrm{~mL}$ of sterile saline solution at each time point. Day of enrollment was considered as $\mathrm{d} 1$, and the study duration was $10 \mathrm{~d}$. Insulin concentrations and whole-body glucose disappearance were evaluated by an i.v. glucose tolerance test conducted at $12 \mathrm{~h}$ and $7 \mathrm{~d}$ following the last rbIL-8 injection. Rectal temperature and blood samples were collected on d 1, 2, 3, and 4 at -30 (before treatment, $0830 \mathrm{~h}$ ), 30, 60, 120, 240, and $360 \mathrm{~min}$ relative to treatment, and daily at $0830 \mathrm{~h}$ for the rest of the study period. Serum was harvested, and the following parameters were measured: $\beta$-hydroxybutyrate (BHB), nonesterified fatty acids, glucose, insulin, plasma urea nitrogen, haptoglobin, and differential blood count. Significant differences were considered when $P \leq 0.05$ and a trend if $0.05<P \leq 0.10$. Serum glucose levels and glucose area under the curve (AUC) did not differ between treatment groups in response to the glucose challenge. However, calves treated with rbIL-8 had

Received January 19, 2019.

Accepted July 10, 2019.

*Corresponding author: rcb28@cornell.edu greater serum insulin concentration and insulin AUC compared with controls. Administration of rbIL-8 increased rectal temperature (rbIL- $8=39.3 \pm 0.1$; CTR $=38.9 \pm 0.1^{\circ} \mathrm{C} ; \pm$ standard error), BHB concentrations $($ rbIL-8 $=3.54 \pm 0.10 ;$ CTR $=2.99 \pm 0.12 \mathrm{mg} / \mathrm{dL})$, counts of lymphocytes (rbIL- $8=4.52 \pm 0.12$; CTR $=$ $3.84 \pm 0.14 \times 10^{3} / \mu \mathrm{L}$ ), monocytes (rbIL-8 $=0.87 \pm$ $0.03 ; \mathrm{CTR}=0.67 \pm 0.04 \times 10^{3} / \mu \mathrm{L}$ ), and granulocytes $\left(\right.$ rbIL- $8=3.54 \pm 0.22 ;$ CTR $\left.=2.66 \pm 0.24 \times 10^{3} / \mu \mathrm{L}\right)$. We conclude that rbIL- 8 induces insulin resistance in Holstein bull calves, accompanied by systemic inflammation and altered blood metabolites and white blood cell populations.

Key words: chemokine, insulin sensitivity, inflammation

\section{INTRODUCTION}

High-producing dairy cows undergo extreme metabolic adaptations during the transition from late gestation to early lactation. Energy demand for milk production increases faster than energy intake during the first 6 to 8 weeks after parturition; thus, high-producing dairy cows experience a period of energy deficit (Bell, 1995; Reynolds et al., 2003). During this period of negative nutrient balance, homeorhetic mechanisms trigger the mobilization of body reserves to support milk synthesis. Transient altered insulin action allows glucose to be spared by peripheral tissues and directed toward the synthesis of lactose in the mammary gland (Bell and Bauman, 1997; De Koster and Opsomer, 2013).

Recently, we demonstrated that a single intrauterine administration of recombinant bovine interleukin- 8 (rbIL-8) to Holstein cows on the day of parturition is associated with an increase in milk production during the first 6 mo of lactation (Zinicola et al., 2019). In that study, intrauterine treatment altered IL-8 plasma levels, suggesting that the increase in milk yield was caused by a systemic effect of rbIL-8. Interleukin- 8 has been shown to induce insulin resistance via inhibition of the AKT pathway (Kobashi et al., 2009). Additionally, 
$24 \mathrm{~h}$ after treatment, we noted a sharp increase in the concentration of serum fatty acids in cows treated with rbIL-8 (Zinicola et al., 2019). It is well documented in the literature that insulin has both anti-lipolytic and lipogenic effects, resulting in reduced fatty acid mobilization (Herdt, 2000; De Koster and Opsomer, 2013). Therefore, the increase in fatty acid levels that we observed could reflect a state of insulin resistance. Interestingly, that increase of circulating fatty acids was not associated with metabolic disorders such as hyperketonemia or clinical ketosis (Zinicola et al., 2019).

Numerous studies have suggested a key role of proinflammatory cytokines, such as IL-8, IL- 6 , and TNF- $\alpha$, in mediating insulin resistance (Kushibiki et al., 2000; Kobashi et al., 2009; Hardy et al., 2011). For instance, Holstein steers treated s.c. once daily for $12 \mathrm{~d}$ with rbTNF- $\alpha$ displayed decreased peripheral insulin sensitivity (Kushibiki et al., 2000). A direct effect of IL-8 on the insulin response has only been demonstrated in vitro, where exposure to IL-8 induced insulin resistance in human adipocytes (Kobashi et al., 2009). Insulin resistance is one of the major homeorhetic regulations that occurs in dairy cows to support lactation. Thus, adjusting energy partitioning by altering tissue responses to insulin has the potential to increase milk production.

Metabolic tests, such as intravenous glucose tolerance test (IVGTT) and intravenous insulin tolerance test, as well as several indices for the estimation of insulin resistance developed for human medicine, have commonly been used in dairy cows to measure insulin sensitivity (Schoenberg and Overton, 2011; Schulz et al., 2014; Mann et al., 2016b). However, in lactating cows, more than $80 \%$ of the glucose produced is drained to the insulin-independent mammary gland (Bell, 1995). Thus, using lactating cows to measure insulin resistance might not be a precise model. Additionally, performing the aforementioned metabolic tests on large-scale studies is not viable due to high costs. However, insulin sensitivity might be higher in calves at the early stages of life, to maintain an anabolic state to support growth.

Previous studies have successfully used pre-weaned calves as an experimental model to evaluate glucose metabolism (Bossaert et al., 2009; Monteiro et al., 2016). For instance, it has been demonstrated that peripheral tissue insulin response differs between beef and dairy cattle (Bossaert et al., 2009). In that study, Holstein-Friesian calves (dairy calves) were found to have lower insulin sensitivity compared with beef-breed calves, suggesting that the reduction in insulin sensitivity in the former may be a consequence of genetic selection for milk yield to support lactation (Bossaert et al., 2009). In the study conducted by Monteiro et al. (2016), calves born from heat-stressed cows without heat abatement were found to have enhanced insulin resistance compared with calves born from heat-stressed cows with heat abatement.

Thus, to initially explore the potential effects of rbIL-8 treatment on bovine glucose metabolism, we hypothesized that systemic administration of rbIL-8 to Holstein bull calves affects response to a glucose challenge, increases systemic inflammation, and alters white blood cell (WBC) populations. Therefore, our objectives were to explore the effects of rbIL- 8 on the insulin response in peripheral tissue, on metabolites, on inflammatory parameters, and on WBC counts in Holstein bull calves.

\section{MATERIALS AND METHODS}

\section{Ethics Statement}

The research protocol was reviewed and approved by the Cornell University Institutional Animal Care and Use Committee (protocol number 2016-0017). The methods were carried out in accordance with the approved guidelines.

\section{Animals, Facilities, and Management}

Eighteen Holstein bull calves between 17 and $29 \mathrm{~d}$ old and between 39.5 and $73.3 \mathrm{~kg}$ of BW were purchased from a commercial dairy farm and shipped to the research barn situated in the large animal sector of Cornell University, Ithaca, NY. Only calves born via unassisted calving were included in the study. After calving, colostrum from primiparous and multiparous cows was pooled and refrigerated. Calves were fed with $4 \mathrm{~L}$ of pooled pasteurized colostrum $\left(60^{\circ} \mathrm{C}\right.$ for $60 \mathrm{~min}$; T-300; Goodnature Products Inc., Orchard Park, NY) with Brix reading $>23 \%$ by esophageal tube (Oral Calf Feeder Bag with Probe, Jorvet, Loveland, CO) within $6 \mathrm{~h}$ of birth. Each individual pen (concrete walls with stainless steel gate; $2 \mathrm{~m}^{2}$ ) was bedded with pine shavings and cleaned on a daily basis. Animals were acclimated for $7 \mathrm{~d}$ before initiation of the study. Calves were fed with $9 \mathrm{~L}$ of raw milk twice daily (0630 and $1600 \mathrm{~h}$ ). Raw milk was purchased from the Cornell University Teaching Dairy. Additionally, water was available between feedings, and calf starter (Calf Starter 18\% CP, DuMOR) was offered ad libitum.

\section{Experimental Design and Sample Collection}

The day of enrollment was considered as d 1, and the study duration was $10 \mathrm{~d}$. Calves were randomly 
allocated to 1 of 2 treatment groups: the rbIL- 8 group (rbIL-8, $\mathrm{n}=10)$ or control (CTR, $\mathrm{n}=8$ ). Originally the study was designed to administer treatments s.c. However, following the first treatment, a large local inflammatory reaction was observed in the calves treated with rbIL-8, so the route of administration was changed to i.v. Therefore, calves assigned to the rbIL- 8 group received 1 s.c. injection (d 1, 0900 h) and 6 i.v. injections (d 1 at $1600 \mathrm{~h} ; \mathrm{d} 2$ and 3 at 0900 and $1600 \mathrm{~h}$; and $\mathrm{d} 4$ at $0900 \mathrm{~h}$ ) of rbIL-8 $(4 \mu \mathrm{g} / \mathrm{kg}$ of BW). Calves assigned to the CTR group received $2 \mathrm{~mL}$ of sterile saline solution (Vedco Inc., Saint Joseph, MO) according to the above method of injection and schedule. Recombinant bovine IL- 8 was produced and purified according to previously described methods (Bicalho et al., 2019). A blood sample was collected on d 2 of life for IgG quantification. Long-term 16Ga catheters (Mila International Inc., Florence, KY) were inserted into the left jugular vein $1 \mathrm{~d}$ before the first treatment, for collection of blood during the study period and for administration of glucose. Before catheterization, calves' hair was clipped on the left side of the neck. The skin was disinfected thrice with iodine $(7.5 \% \mathrm{vol} / \mathrm{vol}$; VetOne, Boise, ID) and once with ethanol $(70 \% \mathrm{vol} / \mathrm{vol}$; VetOne, Boise, ID). Moreover, the skin area where the catheter was placed was sprayed with ethanol once daily during the study period and bandaged. Catheters were maintained by flushing with heparinized saline solution. Blood samples were collected on d 1,2,3, and 4 before treatment; at $30 \mathrm{~min}$ before treatment $(0830 \mathrm{~h})$ and at 30 , $60,120,240$, and 360 min after treatment; and daily at $0830 \mathrm{~h}$ for the rest of the study period, via jugular vein catheter into Vacutainer K2-EDTA Blood Collection Tubes (BD Vacutainer, Franklin Lakes, NJ) and immediately placed on ice. Within $1 \mathrm{~h}$ after blood collection, samples were used for hemogram determination using a veterinary hematology analyzer (HemaTrue, Heska Corp., Loveland, CO), and plasma was obtained by centrifugation $\left(2,000 \times g\right.$ for $15 \mathrm{~min}$ at $\left.4^{\circ} \mathrm{C}\right)$, aliquoted into 2-mL Eppendorf tubes, and stored at $-80^{\circ} \mathrm{C}$ until analysis. Additionally, rectal temperature (RT) was recorded daily and after treatment at the same time as the blood sample collections, as described above. Measurements of BW were taken at birth and on d 0 of the study, at 1400 to $1500 \mathrm{~h}$.

\section{Evaluation of the Peripheral Response to Insulin and Glucose Disappearance}

Whole-body insulin action was evaluated using IVGTT in all calves enrolled in the study. The tests were performed $7 \mathrm{~h}$ after the last treatment $(\mathrm{d} 4,1600$ h) and on d 10 (0800 h) of the study. Calves were fasted for $10 \mathrm{~h}$ before each test. Calves were infused with 0.25 $\mathrm{g} / \mathrm{kg}$ of BW of glucose (dextrose $50 \%$, wt/vol; Phoenix Scientific Inc., St. Joseph, MO) followed by $5 \mathrm{~mL}$ of sterile saline solution to flush the catheter. Blood was sampled at $-15,0,15,30,45,60,90$, and 120 min relative to glucose infusion. Catheters were flushed between sampling points with sterile saline solution containing sodium heparin, to avoid clotting. The initial $3 \mathrm{~mL}$ of blood drawn from the catheter at every sampling point was discarded before the sample was collected to avoid dilution. Blood was collected into Vacutainer K2-EDTA Blood Collection Tubes (BD Vacutainer). Samples were placed on ice immediately and centrifuged at 2,000 $\times g$ for $15 \mathrm{~min}$ at $4^{\circ} \mathrm{C}$ within $1 \mathrm{~h}$ of collection. Plasma was harvested and stored at $-80^{\circ} \mathrm{C}$ until assayed.

\section{Hormone and Metabolite Assays}

Plasma concentrations of fatty acids (NEFA-C kit; Wako Pure Chemical Industries, Richmond, VA), BHB (Sigma-Aldrich, St. Louis, MO; Williamson and Mellanby, 1974), plasma urea nitrogen (PUN; SigmaAldrich), and glucose (Sigma-Aldrich) were determined by colorimetric methods. Haptoglobin concentration was determined using a colorimetric procedure as previously described (Bicalho et al., 2014). Results of haptoglobin concentrations were reported as optical density readings at $450 \mathrm{~nm}$ of wavelength. Insulin concentration was determined using a commercial ELISA kit for bovine insulin (Bovine Insulin ELISA, ALPCO, Salem, $\mathrm{NH}$ ). Following the procedures previously described, a double RIA was used for measuring the plasma concentrations of IGF-1 (Butler et al., 2003, 2004) and growth hormone (GH; Plaut et al., 1987; Rosemberg et al., 1989). An h-IGF-1 receptor grade (lot number EBBCO1) from Gropep (Adelaine, Australia) was used for iodination and standards. Primary antibody anti-hIGF1-rabbit (\#AFP4892898) was obtained from A. F. Parlow at the National Hormone and Pituitary Program (Torrance, CA) and diluted 1:55k for the assay. For the bGH assay, standards and iodinated tracer were made from Upjohn Growth Hormone, Lot number 12, code number 77-001. We added NIDDK-anti-oGH-2 (rabbit) AFPC0123080 at 1:35k as the primary antibody. For both assays the second antibody used for precipitation was sheep anti-rabbit gamma globulin supplied by $\mathrm{W}$. R. Butler, Cornell University. Endotoxin (liposaccharide) levels from purified rbIL-8 were measured via chromogenic assay (Pierce Chromogenic Endotoxin Quant Kit, Thermo Fisher Scientific, Waltham, MA) following manufacturer's instructions. Endotoxin levels 
were less than $5 \mathrm{EU} / \mathrm{mL}$ (data not shown). A total of $2 \mathrm{~mL}$ of purified rbIL- 8 was used for treatments. Thus, calves treated with rbIL- 8 received a solution containing endotoxin levels under the FDA limit of 5 $\mathrm{EU} / \mathrm{kg}$ (FDA, 1985). Plasma level of IgG was measured using a radial immunodiffusion assay according to kit instructions (Bethyl Laboratories Inc., Montgomery, TX). The intraassay coefficient of variation for plasma IgG was $3.0 \%$.

\section{Statistical Methods}

All statistical analyses were performed using SAS (version 9.4; SAS/STAT, SAS Institute Inc., Cary, NC). The effects of rbIL- 8 on blood cell parameters, $\mathrm{RT}$, and plasma concentration of haptoglobin, metabolites, insulin, GH, and IGF-1 were analyzed using mixed general linear mixed models with the MIXED procedure of SAS. Normality and homoscedasticity of residuals were assessed using residual plots. Fixed effects of treatment, time, BW at d 0 , age at $\mathrm{d} 0$, and the 2-way interaction treatment by time were offered to the model as independent variables, and calf was considered a random effect. Manual backward stepwise elimination of variables was undertaken when $P>0.15$. Baseline values (samples collected before treatment on study d 1) for each variable were included in the model as covariates. Differences in serum IgG levels between treatment groups were evaluated using the MIXED procedure of SAS. Several covariance structures were tested (unstructured, autoregressive 1, and compound symmetry), and that with the lowest Akaike information criterion was selected. Plasma concentrations of glucose and insulin from IVGTT were used to create response curves. The positive incremental area under the curve (AUC) of glucose and insulin was calculated in SAS, correcting for baseline, based on the trapezoidal method as previously described (Cardoso et al., 2011). Significant differences were considered when $P \leq 0.05$ or a trend if $0.05<P \leq 0.10$. For all models, significant differences between time points were tested using Bonferroni adjustment for multiple comparisons. Data are reported as LSM \pm SEM unless otherwise stated.

\section{RESULTS}

\section{Descriptive Results}

No differences $(P>0.65)$ in $\mathrm{BW}$ at birth, BW at study d 0 , or age at study d 0 were detected between treatment groups. No differences were detected in IgG concentration between treatment groups $(\mathrm{CTR}=12.6$ $\pm 0.07, \mathrm{rbIL}-8=12.5 \pm 0.06 \mathrm{~g} / \mathrm{L} ; P=0.69)$.

\section{Intravenous Glucose Tolerance Test}

Glucose AUC did not differ between treatment groups among all IVGTT (Figure 1A, B). For the IVGTT performed at $12 \mathrm{~h}$ after treatment, plasma concentration of insulin was greater in the rbIL-8 group at $15(P=$ $0.03)$ and $30(P=0.01)$ min after glucose infusion and tended to be greater $(P=0.08)$ at 45 min compared with the CTR group (Figure 1A). For the IVGTT performed on study d 10, rbIL-8 calves had higher plasma concentrations of insulin at $15(P=0.001)$ and $30(P$ $=0.01)$ min after glucose infusion compared with their CTR counterparts (Figure 1B). Compared with CTR calves, rbIL-8 calves tended to have a greater insulin AUC during IVGTT conducted $12 \mathrm{~h}$ after treatment $(P$ $=0.06)$ and had greater $(P=0.002)$ insulin AUC during IVGTT performed on study d 10 (Figure 1A, B).

\section{Hormone and Metabolite Concentrations}

No differences were observed in the plasma concentrations of glucose, insulin, GH, or IGF-1 between treatment groups (Figures 2 and 3). Treatment with rbIL-8 increased $(P=0.006)$ BHB plasma concentrations particularly at study d 3 and 8 (Figure 2). No differences were observed in plasma concentrations of fatty acids between the 2 groups (Figure 2).

Figure 4 illustrates the dynamics of insulin, glucose, $\mathrm{BHB}$, and fatty acids after treatment on $\mathrm{d} 1$ (s.c.) and d 2 (i.v.). After the s.c. treatment, no differences were observed for serum concentrations of glucose, insulin, BHB, or fatty acids between treatment groups. However, following the i.v. treatment, the administration of rbIL-8 did alter serum levels of glucose $(P=0.09)$, insulin $(P=0.06)$, BHB $(P=0.07)$, and fatty acids $(P=$ 0.10). Compared with CTR calves, calves treated with rbIL-8 had a higher $(P=0.05)$ glucose concentration at $30 \mathrm{~min}$ after the first treatment on $\mathrm{d} 2$, followed by an acute increase $(P=0.001)$ in insulin concentrations at $120 \mathrm{~min}$. Plasma BHB concentration tended to be greater $(P=0.09)$ on $\mathrm{d} 2$ at $30 \mathrm{~min}$, and increased from $60(P=0.04)$ to $120(P=0.01)$ min after treatment in the rbIL-8 group compared with controls. Finally, we observed higher $(P=0.03)$ concentrations of fatty acids at 360 min on $\mathrm{d} 2$ in calves that were treated with rbIL-8 compared with controls.

Although an overall effect of treatment on plasma concentrations of PUN was not observed, an interaction between treatment and time was observed $(P=0.004)$. Calves treated with rbIL-8 had higher $(P=0.002)$ concentrations of PUN in plasma on d 10 compared with controls (Figure 2). Treatment by time interaction was also observed $(P=0.07)$ for haptoglobin concentra- 
- rblL-8 $\rightarrow$ Controls
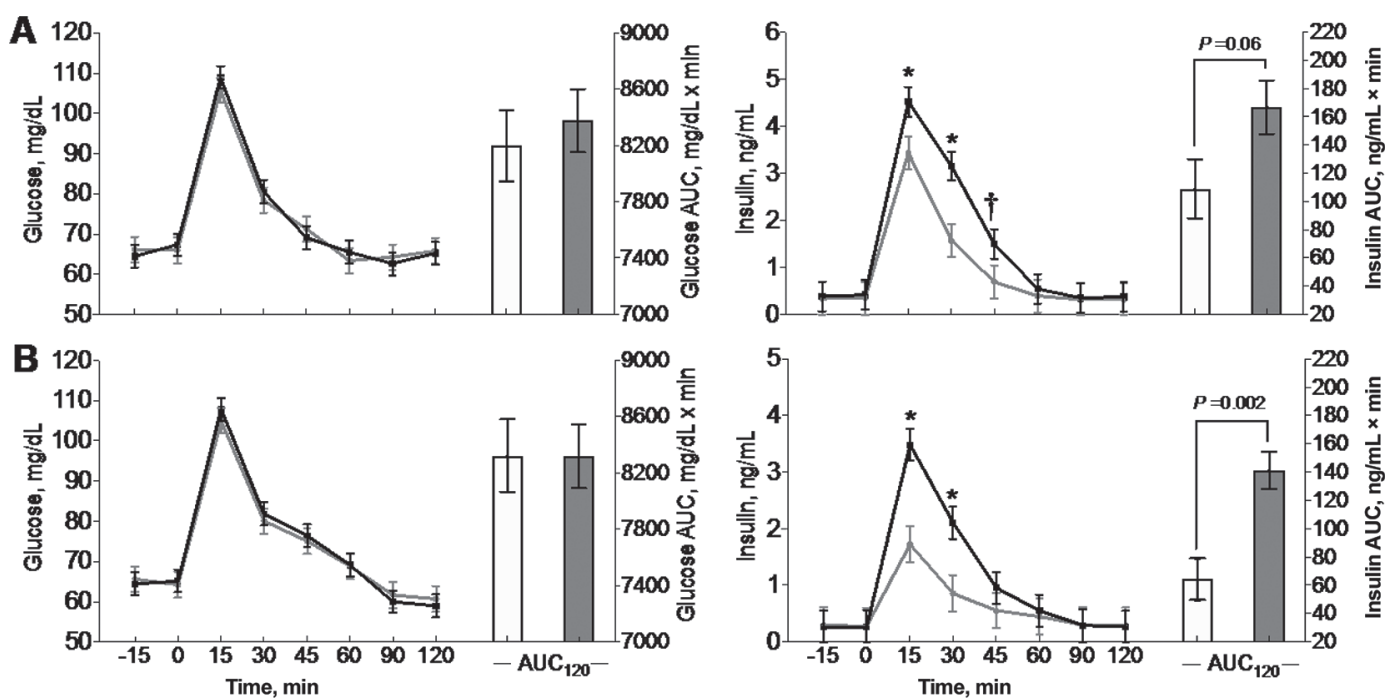

Figure 1. Glucose and insulin responses to the glucose tolerance test in Holstein bull calves treated with recombinant bovine interleukin- 8 (rbIL-8; black lines with squares) or control calves (gray lines with circles) at $12 \mathrm{~h}(\mathrm{~A})$ and $7 \mathrm{~d}$ (B) after treatments. White bars and dark gray bars represent the area under the curve [AUC; $\mathrm{mg} / \mathrm{dL}$ (glucose) and $\mathrm{ng} / \mathrm{mL}$ (insulin) per $120 \mathrm{~min}$ ] of control and rbIL- 8 calves, respectively. ${ }^{*} P$ $\leq 0.05 ; \dagger P \leq 0.1$. Results are presented as LSM \pm SEM.

tions, as calves treated with rbIL-8 had higher concentrations of haptoglobin in plasma on $\mathrm{d} 2(P=0.02)$ and d $3(P=0.002)$ compared with controls (Figure 2$)$.

\section{Hemogram Parameters and Rectal Temperature}

Results of daily, multi-time point monitoring of RT, and absolute numbers of WBC, lymphocytes (LYM), monocytes (MONO) and granulocytes (GRAN) are depicted in Figures 5 and 6 . Treatment with rbIL-8 affected RT $(P=0.03)$, WBC $(P=0.005)$, LYM $(P=$ $0.0007)$, MONO $(P=0.0007)$, and GRAN $(P=0.01)$. Calves treated with rbIL-8 had increased RT at $60(P$ $=0.02)$ and $120(P=0.01)$ min after the first treatment on $\mathrm{d} 2$, and a trend $(P=0.06)$ was observed 30 min after treatment on $\mathrm{d} 3$. Moreover, RT was higher at $\mathrm{d} 8(P=0.01)$ and $\mathrm{d} 10(P=0.01)$, and tended to be higher at d $9(P=0.06)$ in the rbIL8-treated group compared with the CTR group (Figure 5). Calves in the rbIL-8 treatment group had $(P=0.001)$ and tended to have $(P=0.07)$ higher WBC counts at 240 and 360 min after the first treatment on $\mathrm{d} 1$, and at $30 \mathrm{~min}$ before the first treatment on d 2, respectively (Figure $6)$. Compared with controls, rbIL-8 calves tended to have $(P=0.08)$ higher LYM counts at 30 min after the first treatment on d 2 and at $30 \mathrm{~min}$ before the first treatment on $\mathrm{d} 4$, and a significant increase $(P=0.01)$ was observed on d 10 (Figure 6). Relative to controls, rbIL-8 calves tended to have $(P=0.06)$ and had higher $(P=0.03)$ counts of MONO at 240 and 360 min after the first treatment on d 1, respectively. Additionally, a trend $(P=0.07)$ was observed at 120 min after the first treatment on d 4, at which point rbIL-8 calves had higher MONO concentration than controls (Figure 6). Finally, higher $(P=0.001)$ counts of GRAN were observed at 240 and $360 \mathrm{~min}$ after the first treatment on $\mathrm{d} 1$ in the treatment group compared with the controls (Figure 6).

\section{DISCUSSION}

Numerous studies have suggested that interleukin- 8 is associated with insulin resistance (Kobashi et al., 2009; Hardy et al., 2011). However, it is unknown whether rbIL-8 treatment in Holstein animals can induce insulin resistance. Therefore, to initially explore the potential effects of rbIL- 8 treatment on bovine metabolism, particularly on insulin action, the present study hypothesized that systemic administration of rbIL-8 would reduce peripheral insulin sensitivity. Insulin concentrations and whole-body glucose disappearance were evaluated through 2 IVGTT, at $12 \mathrm{~h}$ and $7 \mathrm{~d}$ following the last rbIL-8 injection. In accordance with our hypothesis, systemic treatment with rbIL-8 induced peripheral insulin resistance in Holstein bull calves. In addition, rbIL-8 administration resulted in elevated RT, increased haptoglobin, and altered blood metabolites and WBC counts.

In an accompanying article, we demonstrated that a single intrauterine administration of rbIL- 8 treatment 


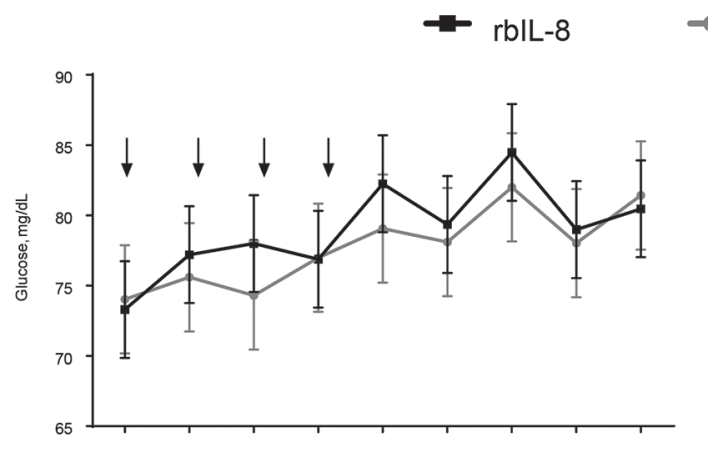

$\rightarrow$ Controls
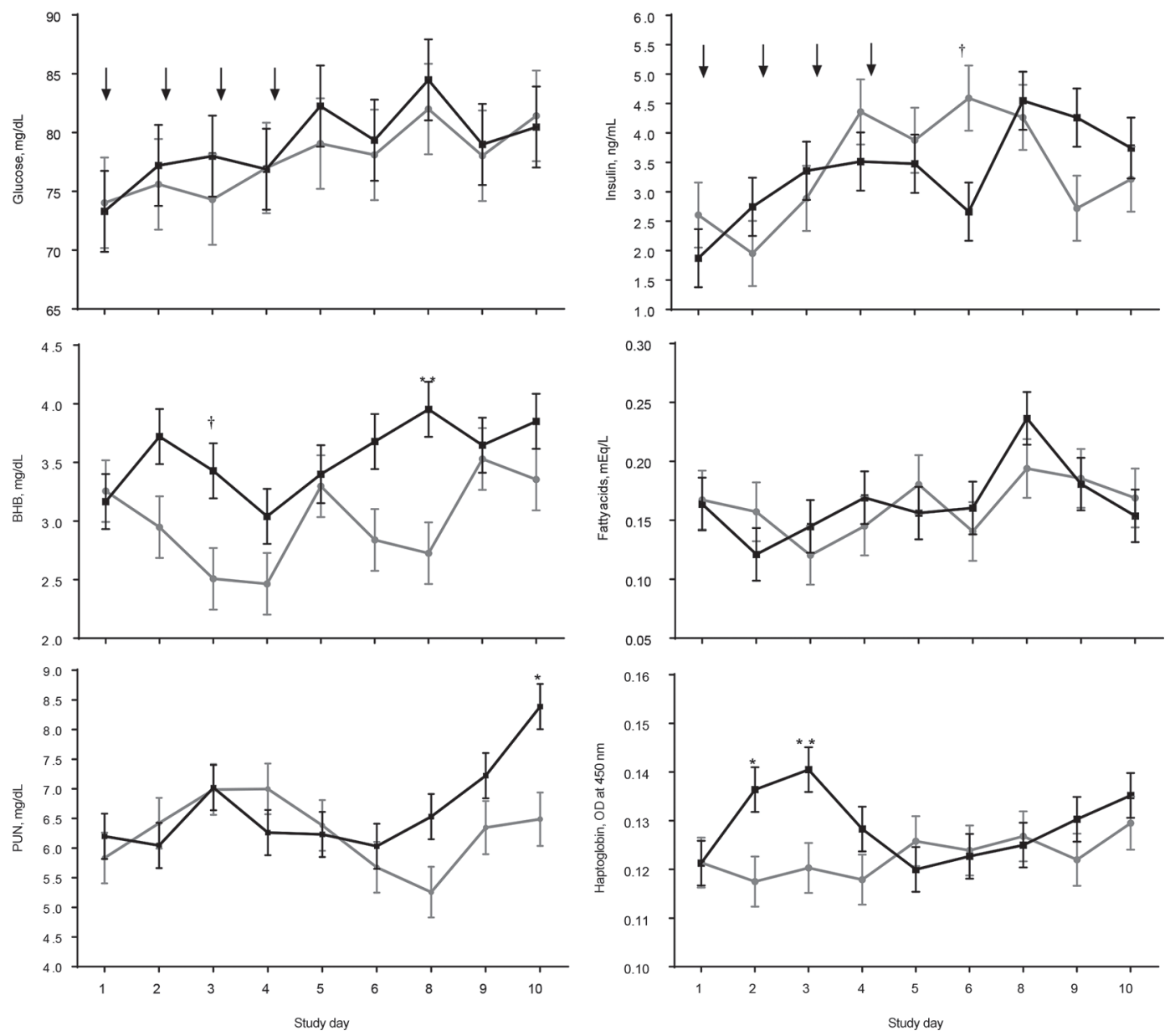

Figure 2. Plasma concentrations of glucose, insulin, BHB, fatty acids, plasma urea nitrogen (PUN), and haptoglobin (optical density units, OD) from d 1 to 10 of the study of Holstein bull calves treated with recombinant bovine interleukin-8 (rbIL-8; black lines with squares) and control calves (gray lines with circles). Arrows indicate times of treatments. ${ }^{* *} P \leq 0.01 ;{ }^{*} P \leq 0.05 ; \dagger P \leq 0.10$. Results are presented as LSM \pm SEM.

shortly after parturition increased milk yield during the first 6 mo of lactation in Holstein cows (Zinicola et al., 2019). Although the cellular mechanism underlying the decrease in insulin response in early lactation is not clear, in that study, our data suggested that rbIL-8 might induce insulin resistance. In the present study, a metabolic test (IVGTT) showed that systemic treatments with rbIL-8 induced insulin resistance in Holstein bull calves. Additionally, we showed that after the first treatment on d 2, rbIL-8 induced hyperglycemia that was reflected by an increase in serum insulin. In animals that are not insulin-resistant, insulin has antilipolytic and lipogenic effects. However, in our study, instead of identifying a depletion in fatty acids, we observed that calves treated with rbIL- 8 had a higher serum concentration of fatty acids (coincident with insulin elevation) compared with controls. Considering the drastic differences in metabolism between calves and lactating cows, extrapolation of insulin resistance results following rbIL-8 treatment presented in the current study to postpartum cows during early lactation should be done with caution. Therefore, studies using lactating cows are needed to assess whether increased insulin resistance may be expected following rbIL-8 administration.

In mammals, the role of pro-inflammatory cytokines such as IL-8, IL-6, and TNF- $\alpha$ in altering the insulin response has been studied (Kushibiki et al., 2000; Kobashi et al., 2009; Hardy et al., 2011). For instance, Holstein steers treated once daily for $12 \mathrm{~d}$ with $\mathrm{rbTNF}-\alpha$ exhibited decreased peripheral insulin sensitivity when assessed with IVGTT and an intravenous insulin tolerance test (Kushibiki et al., 2000). Although, to our knowledge, a direct effect of IL-8 on insulin action in 
- rblL-8 $\rightarrow$ Controls
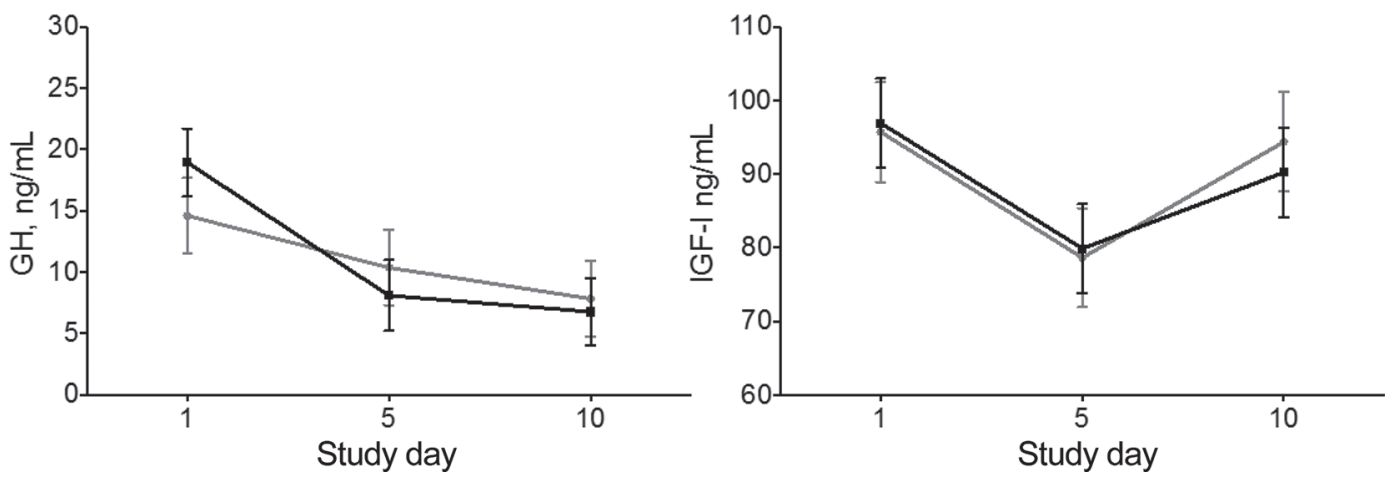

Figure 3. Plasma concentrations of growth hormone (GH) and IGF-1 at d 1, 5, and 10 of the study of recombinant bovine IL-8 (rbIL-8)treated and control calves. Results are presented as LSM \pm SEM.

dairy cattle has not been investigated, the results presented here for rbIL-8 on the development of insulin resistance are in accordance with the human literature. The expression of IL- 8 in omental fat depots is 2.7 -fold greater in obese humans who are insulin-resistant, compared with obese patients classified as insulin-sensitive (Hardy et al., 2011). In support, the direct effect of IL-8 on the response to insulin was demonstrated in vitro, wherein exposure to IL-8 induced insulin resistance in human adipocytes by reducing the effect of the insulinstimulated AKT pathway (Kobashi et al., 2009).
Insulin-receptor signaling has a direct effect on activation of the AKT pathway, which promotes GLUT4 translocation to the cell membrane for glucose uptake, stimulates glycogen synthesis, and inhibits gluconeogenesis (Brockman, 1985; Stephens and Pilch, 1995; Barthel and Schmoll, 2003). In addition, the AKT pathway plays a central role in muscle hypertrophy and atrophy (Sandri, 2008). Its activation decreases muscle proteolysis by inhibiting the ubiquitin-proteasome pathway, which results in decreased muscle mobilization (Mann et al., 2016a). Although PUN can be influenced by a

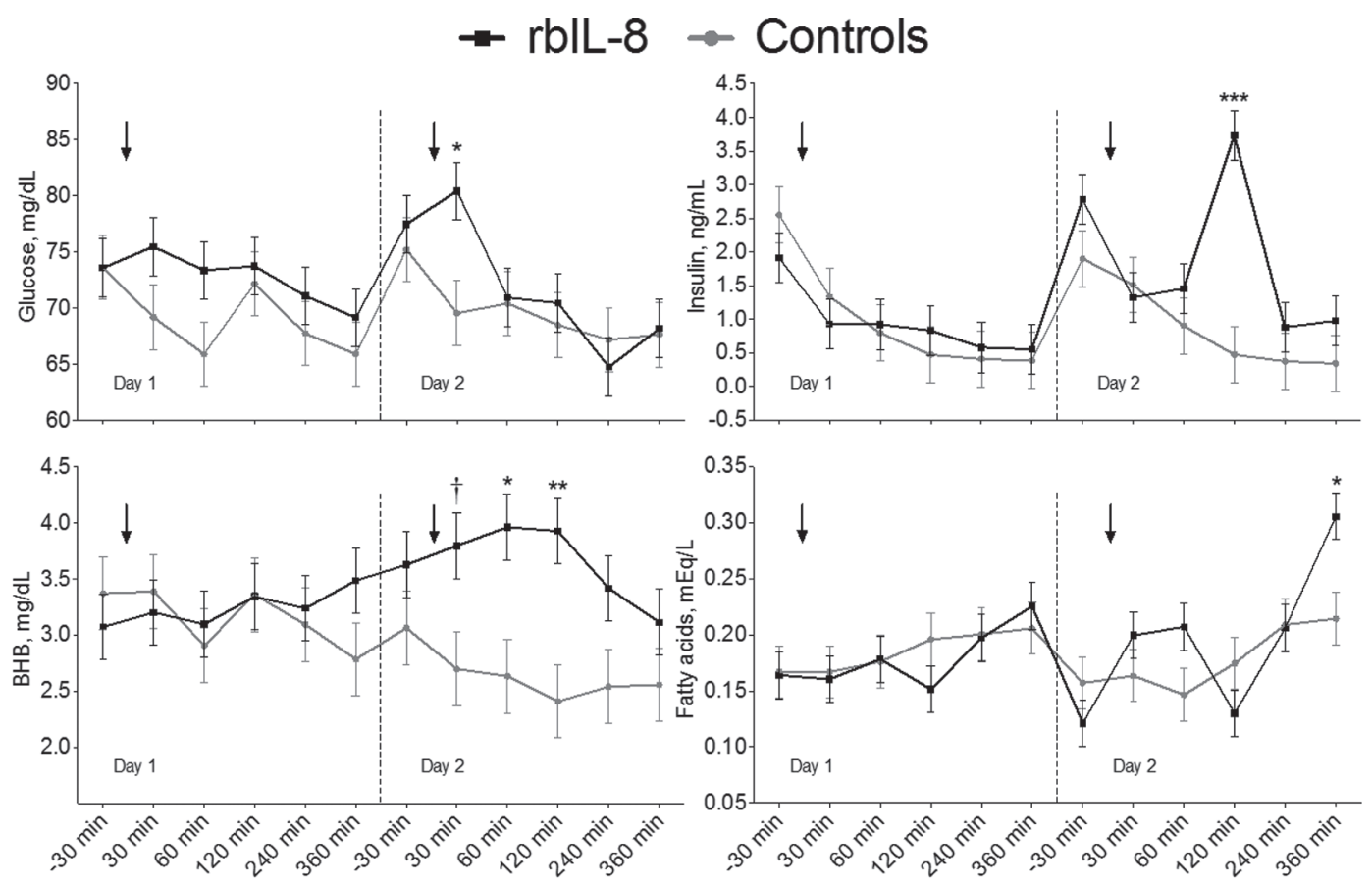

Figure 4. Dynamics of plasma concentrations of glucose, insulin, BHB, and fatty acids after the first treatment (0900 h) on d 1 and 2 of the study of Holstein bull calves treated with recombinant bovine interleukin-8 (rbIL-8; black lines with squares) and control calves (gray lines with circles). Arrows indicate times of treatments. ${ }^{* *} P \leq 0.001 ;{ }^{* *} P \leq 0.01 ;{ }^{*} P \leq 0.05 ; \dagger P \leq 0.1$. Results are presented as LSM $\pm \mathrm{SEM}$. 


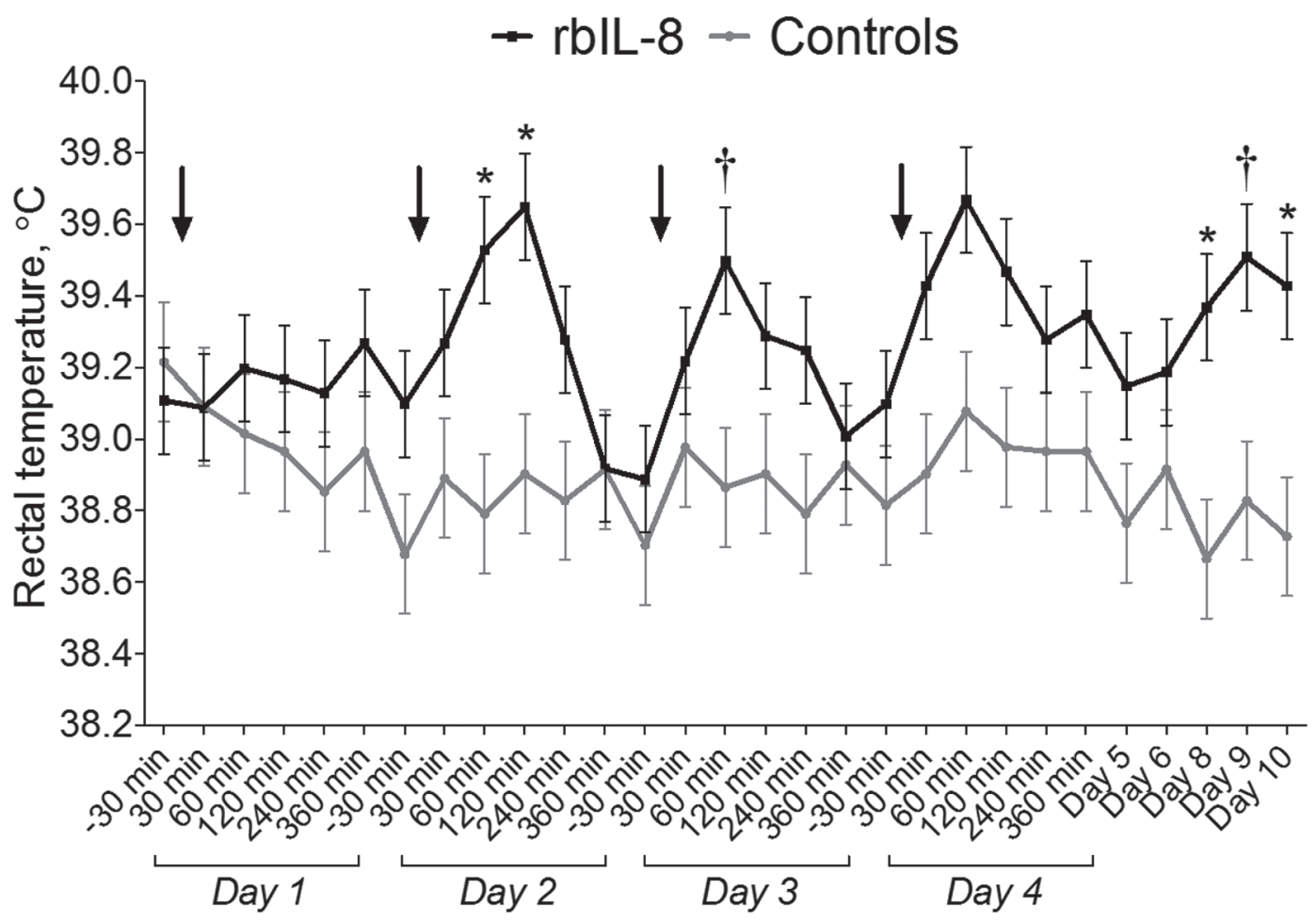

Figure 5. Dynamics of rectal temperature following the first treatment (0900 h) on d 1,2,3, and 4 of the study of Holstein bull calves treated with recombinant bovine interleukin-8 (rbIL-8; black lines with squares) and control calves (gray lines with circles). Arrows indicate times of treatments. ${ }^{*} P \leq 0.05 ; \dagger P \leq 0.1$. Results are presented as $\mathrm{LSM} \pm \mathrm{SEM}$.

variety of parameters (e.g., dehydration, dietary protein and energy intake, rumen degradability, and liver and kidney function), it is used as a blood marker to directly reflect the protein status of an animal. For instance, in fasted steers, increased muscle protein turnover to meet energy needs is associated with higher levels of PUN (Ward et al., 1992). In our study, although an overall treatment effect on plasma PUN was not observed, a treatment by time interaction was observed, because on d 10, rbIL-8-treated calves had a higher concentration of PUN compared with controls.

Considering our clear findings for rbIL-8 on altered insulin action, concomitant with the observed increased serum concentrations of BHB and PUN, it is possible to surmise that the reduced insulin response might led to diminished AKT-phosphorylation (reducing its activity), which could promote the release of glucogenic and ketogenic amino acids from skeletal muscle to the blood stream. Thus, the elevated plasma BHB and PUN levels observed in this study could be due to a systemic increase in amino acid levels owing to increased muscle breakdown. However, in the present study, AKT-phosphorylation in muscle and adipose tissue was not assessed. Moreover, feed intake was not measured in the present study, and this could also have altered BHB and PUN levels. Thus, this hypothesis needs to be further investigated.

In the present study, the repeated administration of rbIL-8 caused several changes to the WBC population. Interleukin-8 is the main chemoattractant for neutrophils and can be produced by smooth muscle, epithelial cells, endothelial cells, and any cell of the innate immune system with toll-like receptors (Mitchell et al., 2003). In addition, cytokines are well-known promoters of WBC differentiation. Here, we did detect a shift in WBC counts (e.g., for LYM, MONO, and GRAN) in calves treated with rbIL-8, a pathology previously linked with obesity-related insulin resistance in humans (Vozarova et al., 2002a,b; Harford et al., 2011). It has been demonstrated that infiltration of macrophages and $\mathrm{T}$ cells into peripheral tissues contributes to inflammation and alters insulin action there (Vozarova et al., 2002a; Kintscher et al., 2008; Harford et al., 2011). In addition, it has been shown that a high WBC count is associated with a decrease in insulin action and predicts the development of type 2 diabetes (Vozarova et al., $2002 b$ ). Based on the effects of rbIL-8 on blood immune cells in the present study, is reasonable to surmise that as a result of an elevated WBC population caused by a direct or indirect effect of rbIL-8 treatment, immune 


\section{$\rightarrow$ rblL-8 $\rightarrow$ Controls}
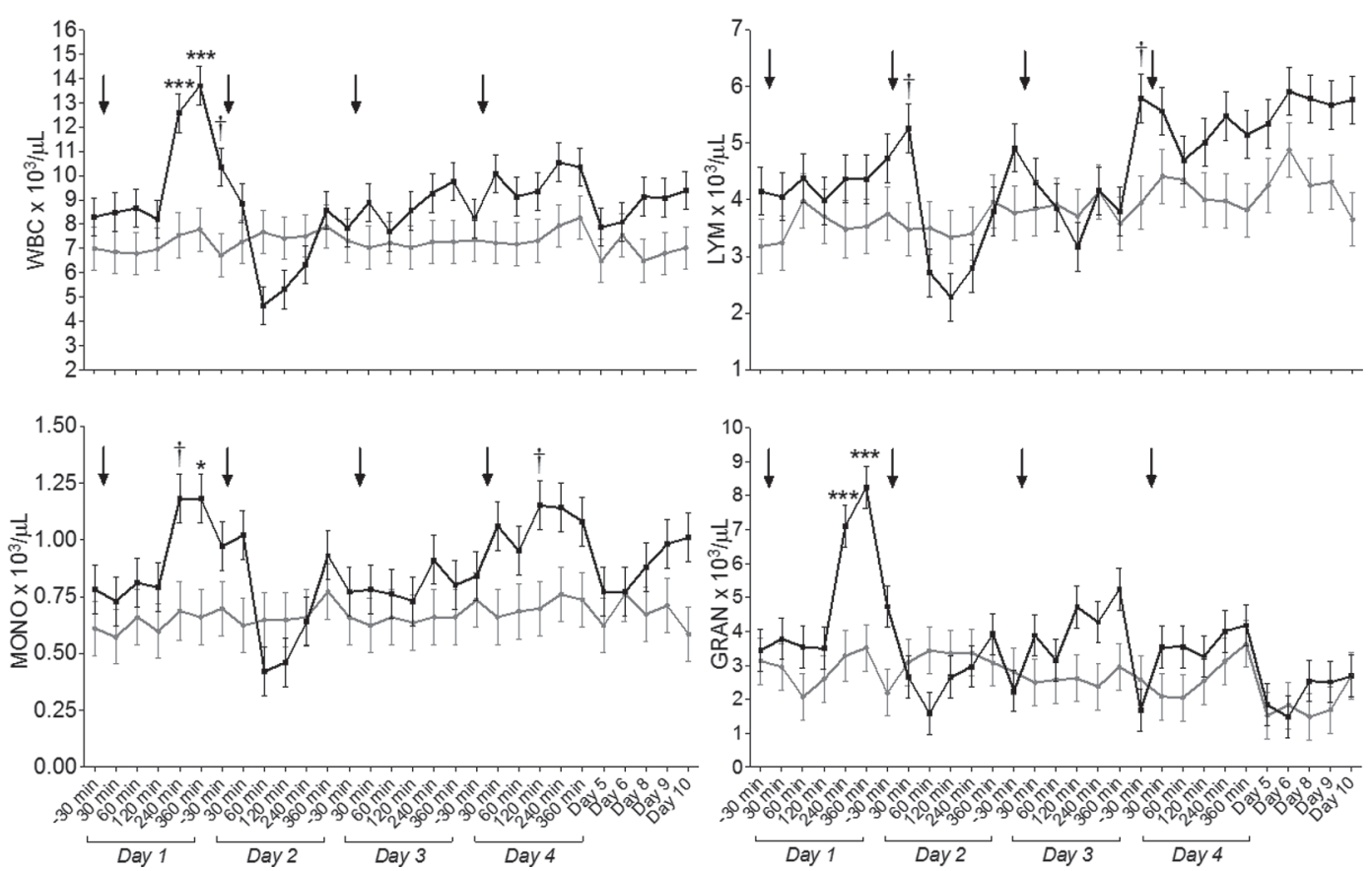

Figure 6. Dynamics of the concentration in absolute numbers of white blood cells (WBC), lymphocytes (LYM), monocytes (MONO), and granulocytes (GRAN) following the first treatment $(0900 \mathrm{~h})$ on d 1,2,3 and 4 of the study of Holstein bull calves treated with recombinant bovine interleukin-8 (rbIL-8; black lines with squares) and control calves (gray lines with circles). Arrows indicate times of treatments. ${ }^{* * *} P \leq$ $0.001 ;{ }^{*} P \leq 0.05 ; \dagger P \leq 0.1$. Results are presented as LSM \pm SEM.

cells might have been accumulated in peripheral tissues, diminishing the insulin response. However, further work needs to be completed using specific techniques to detect target immune cells in blood and peripheral tissues before and after rbIL-8 therapy to elucidate the aforementioned hypothesis.

A marker of inflammation evaluated in the present study was the plasma concentration of a member of acute-phase proteins. Haptoglobin is produced by the liver in response to a systemic increase in pro-inflammatory cytokines (Baumann et al., 1989). Here, we demonstrated that repeated administration of rbIL-8 to bull calves induced a significant increase in plasma haptoglobin during the treatment period. Therefore, considering together the detected increases of haptoglobin, RT, and blood cell counts in calves treated with rbIL- 8 , it is plausible that rbIL-8 elicited those effects by acting as an inflammatory stimulus. Along these lines, inflammation, as indicated by increased serum levels of acute phase proteins, was previously observed in Holstein steers challenged with an i.v. infusion of lipopolysaccharide (Kvidera et al., 2016). Moreover, in that study, challenged animals showed increased circulating levels of insulin, glucose, fatty acids, and BHB compared with controls; similar metabolic changes were observed in the current study following rbIL- 8 treatment. Therefore, it is reasonable to surmise that the alterations to metabolism and insulin action observed in the current study were likely consequences of rbIL-8 treatment inducing an inflammatory response.

Lastly, we explored the effect of rbIL-8 treatment on plasma GH and IGF-1 concentrations. During early lactation, the GH/IGF-1 axis is uncoupled. This uncoupling favors nutrient partitioning and, therefore, increased milk production (Rhoads et al., 2007; Lucy et al., 2009). Cytokines are known to inhibit GH signaling via production of suppressors of cytokine signaling (SOCS) proteins (Rico-Bautista et al., 2006). Thus, we suggested that the observed higher milk yield in cows treated with rbIL- 8 might be a result of IL-8 favoring the uncoupling of the GH/IGF-1 axis (Zinicola et al., 2019). In contrast with our hypothesis, in the present study GH and IGF-1 levels were not affected by rbIL8 treatment. However, the potential effect of rbIL- 8 treatment on GH and IGF-1 concentrations in Holstein cows during early lactation remains in need of further investigation.

In the present study, we demonstrated that rbIL-8 administration alters insulin sensitivity in Holstein bull calves, which potentially involves rbIL-8 exerting 
an effect at an immunological-metabolic interface. It is unknown whether the same response after rbIL-8 treatment would be observed in lactating cows. Future research in dairy cows during early lactation is needed, to characterize the potential role of $\mathrm{rbIL}-8$ in altering the action of insulin.

\section{CONCLUSIONS}

In summary, systemic rbIL-8 administration induces peripheral insulin resistance in Holstein bull calves, and this resistance persisted for $7 \mathrm{~d}$ after treatment, induces systemic inflammation, and alters blood metabolites and blood cell counts. Further research in lactating cows is needed to evaluate the effect of rbIL- 8 on the homeorhetic adaptations that occur after parturition to support lactation, particularly on the development of insulin resistance.

\section{ACKNOWLEDGMENTS}

This work was partially supported by Zoetis Animal Health (Kalamazoo, MI) and by Agriculture and Food Research Initiative Competitive Grant No 201767015-26541 from the United States Department of Agriculture National Institute of Food and Agriculture (Washington, DC).

\section{REFERENCES}

Barthel, A., and D. Schmoll. 2003. Novel concepts in insulin regulation of hepatic gluconeogenesis. Am. J. Physiol. Endocrinol. Metab. 285:E685-E692.

Baumann, H., K. R. Prowse, S. Marinkovic, K. A. Won, and G. P. Jahreis. 1989. Stimulation of hepatic acute phase response by cytokines and glucocorticoids. Ann. N. Y. Acad. Sci. 557:280-295., discussion 295-286.

Bell, A. W. 1995. Regulation of organic nutrient metabolism during transition from late pregnancy to early lactation. J. Anim. Sci. 73:2804-2819

Bell, A. W., and D. E. Bauman. 1997. Adaptations of glucose metabolism during pregnancy and lactation. J. Mammary Gland Biol. Neoplasia 2:265-278.

Bicalho, L. M. S., M. Zinicola, V. S. Machado, F. S. Lima, A. G. V. Teixeira, C. Narbus, M. R. Xavier, H. Higgins, and R. C. Bicalho. 2019. Effects of recombinant bovine interleukin-8 (rbIL-8) treatment on health, metabolism, and lactation performance in Holstein cattle I: Production and functional characterization of rbIL-8 in vitro and in vivo. J. Dairy Sci. 102:10304-10315. https://doi .org/10.3168/jds.2019-16334.

Bicalho, M. L., F. S. Lima, E. K. Ganda, C. Foditsch, E. B. Meira Jr., V. S. Machado, A. G. Teixeira, G. Oikonomou, R. O. Gilbert, and R. C. Bicalho. 2014. Effect of trace mineral supplementation on selected minerals, energy metabolites, oxidative stress, and immune parameters and its association with uterine diseases in dairy cattle. J. Dairy Sci. 97:4281-4295.

Bossaert, P., J. L. Leroy, S. De Campeneere, S. De Vliegher, and G. Opsomer. 2009. Differences in the glucose-induced insulin response and the peripheral insulin responsiveness between neonatal calves of the Belgian Blue, Holstein-Friesian, and East Flemish breeds. J. Dairy Sci. 92:4404-4411.
Brockman, R. P. 1985. Role of insulin in regulating hepatic gluconeogenesis in sheep. Can. J. Physiol. Pharmacol. 63:1460-1464.

Butler, S. T., A. L. Marr, S. H. Pelton, R. P. Radcliff, M. C. Lucy, and W. R. Butler. 2003. Insulin restores GH responsiveness during lactation-induced negative energy balance in dairy cattle: Effects on expression of IGF-I and GH receptor 1A. J. Endocrinol. 176:205-217.

Butler, S. T., S. H. Pelton, and W. R. Butler. 2004. Insulin increases 17 beta-estradiol production by the dominant follicle of the first postpartum follicle wave in dairy cows. Reproduction 127:537-545.

Cardoso, F. C., W. Sears, S. J. LeBlanc, and J. K. Drackley. 2011. Technical note: Comparison of 3 methods for analyzing areas under the curve for glucose and nonesterified fatty acids concentrations following epinephrine challenge in dairy cows. J. Dairy Sci. 94:6111-6115.

De Koster, J. D., and G. Opsomer. 2013. Insulin resistance in dairy cows. Vet. Clin. North Am. Food Anim. Pract. 29:299-322.

FDA (Food and Drug Administration). 1985. Bacterial endotoxin/ pyrogens. Accessed Mar. 20, 2018. https://www.fda.gov/ICECI/ Inspections/InspectionGuides/InspectionTechnicalGuides/ ucm072918.htm.

Hardy, O. T., R. A. Perugini, S. M. Nicoloro, K. Gallagher-Dorval, V. Puri, J. Straubhaar, and M. P. Czech. 2011. Body mass indexindependent inflammation in omental adipose tissue associated with insulin resistance in morbid obesity. Surg. Obes. Relat. Dis. 7:60-67.

Harford, K. A., C. M. Reynolds, F. C. McGillicuddy, and H. M. Roche. 2011. Fats, inflammation and insulin resistance: Insights to the role of macrophage and T-cell accumulation in adipose tissue. Proc. Nutr. Soc. 70:408-417.

Herdt, T. H. 2000. Ruminant adaptation to negative energy balance. Influences on the etiology of ketosis and fatty liver. Vet. Clin. North Am. Food Anim. Pract. 16:215-230.

Kintscher, U., M. Hartge, K. Hess, A. Foryst-Ludwig, M. Clemenz, M. Wabitsch, P. Fischer-Posovszky, T. F. Barth, D. Dragun, T. Skurk, H. Hauner, M. Bluher, T. Unger, A. M. Wolf, U. Knippschild, V. Hombach, and N. Marx. 2008. T-lymphocyte infiltration in visceral adipose tissue: A primary event in adipose tissue inflammation and the development of obesity-mediated insulin resistance. Arterioscler. Thromb. Vasc. Biol. 28:1304-1310.

Kobashi, C., S. Asamizu, M. Ishiki, M. Iwata, I. Usui, K. Yamazaki, K. Tobe, M. Kobayashi, and M. Urakaze. 2009. Inhibitory effect of IL-8 on insulin action in human adipocytes via MAP kinase pathway. J. Inflamm. (Lond.) 6:25.

Kushibiki, S., K. Hodate, Y. Ueda, H. Shingu, Y. Mori, T. Itoh, and Y. Yokomizo. 2000. Administration of recombinant bovine tumor necrosis factor-alpha affects intermediary metabolism and insulin and growth hormone secretion in dairy heifers. J. Anim. Sci. 78:2164-2171.

Kvidera, S. K., E. A. Horst, M. Abuajamieh, E. J. Mayorga, M. V Sanz Fernandez, and L. H. Baumgard. 2016. Technical note: A procedure to estimate glucose requirements of an activated immune system in steers. J. Anim. Sci. 94:4591-4599.

Lucy, M. C., G. A. Verkerk, B. E. Whyte, K. A. Macdonald, L. Burton, R. T. Cursons, J. R. Roche, and C. W. Holmes. 2009. Somatotropic axis components and nutrient partitioning in genetically diverse dairy cows managed under different feed allowances in a pasture system. J. Dairy Sci. 92:526-539.

Mann, S., D. V. Nydam, A. Abuelo, F. A. L. Yepes, T. R. Overton, and J. J. Wakshlag. 2016a. Insulin signaling and skeletal muscle atrophy and autophagy in transition dairy cows either overfed energy or fed a controlled energy diet prepartum. J. Comp. Physiol. B 186:513-525.

Mann, S., F. A. L. Yepes, M. Duplessis, J. J. Wakshlag, T. R. Overton, B. P. Cummings, and D. V. Nydam. 2016b. Dry period plane of energy: Effects on glucose tolerance in transition dairy cows. J. Dairy Sci. 99:701-717.

Mitchell, G. B., B. N. Albright, and J. L. Caswell. 2003. Effect of interleukin-8 and granulocyte colony-stimulating factor on priming and activation of bovine neutrophils. Infect. Immun. 71:1643-1649. 
Monteiro, A. P. A., J.-R. Guo, X.-S. Weng, B. M. Ahmed, M. J. Hayen, G. E. Dahl, J. K. Bernard, and S. Tao. 2016. Effect of maternal heat stress during the dry period on growth and metabolism of calves. J. Dairy Sci. 99:3896-3907. https://doi.org/10.3168/jds .2015-10699.

Plaut, K., D. E. Bauman, N. Agergaard, and R. M. Akers. 1987. Effect of exogenous prolactin administration on lactational performance of dairy cows. Domest. Anim. Endocrinol. 4:279-290.

Reynolds, C. K., P. C. Aikman, B. Lupoli, D. J. Humphries, and D. E. Beever. 2003. Splanchnic metabolism of dairy cows during the transition from late gestation through early lactation. J. Dairy Sci. 86:1201-1217.

Rhoads, R. P., J. W. Kim, M. E. Van Amburgh, R. A. Ehrhardt, S J. Frank, and Y. R. Boisclair. 2007. Effect of nutrition on the GH responsiveness of liver and adipose tissue in dairy cows. J. Endocrinol. 195:49-58.

Rico-Bautista, E., A. Flores-Morales, and L. Fernandez-Perez. 2006. Suppressor of cytokine signaling (SOCS) 2, a protein with multiple functions. Cytokine Growth Factor Rev. 17:431-439.

Rosemberg, E., M. L. Thonney, and W. R. Butler. 1989. The effects of bovine growth hormone and thyroxine on growth rate and carcass measurements in lambs. J. Anim. Sci. 67:3300-3312.

Sandri, M. 2008. Signaling in muscle atrophy and hypertrophy. Physiology (Bethesda) 23:160-170.

Schoenberg, K. M., and T. R. Overton. 2011. Effects of plane of nutrition and 2,4-thiazolidinedione on insulin responses and adipose tissue gene expression in dairy cattle during late gestation. J. Dairy Sci. 94:6021-6035. https://doi.org/10.3168/jds.2011-4533.

Schulz, K., J. Frahm, U. Meyer, S. Kersten, D. Reiche, J. Rehage, and S. Danicke. 2014. Effects of prepartal body condition score and peripartal energy supply of dairy cows on postpartal lipolysis, energy balance and ketogenesis: An animal model to investigate subclinical ketosis. J. Dairy Res. 81:257-266. https://doi.org/10 $.1017 /$ S0022029914000107.

Stephens, J. M., and P. F. Pilch. 1995. The metabolic regulation and vesicular transport of GLUT4, the major insulin-responsive glucose transporter. Endocr. Rev. 16:529-546.

Vozarova, B., N. Stefan, R. Hanson, R. S. Lindsay, C. Bogardus, P. A. Tataranni, C. Metz, and R. Bucala. 2002a. Plasma concentrations of macrophage migration inhibitory factor are elevated in Pima Indians compared to Caucasians and are associated with insulin resistance. Diabetologia 45:1739-1741.

Vozarova, B., C. Weyer, R. S. Lindsay, R. E. Pratley, C. Bogardus, and P. A. Tataranni. 2002b. High white blood cell count is associated with a worsening of insulin sensitivity and predicts the development of type 2 diabetes. Diabetes 51:455-461.

Ward, J. R., D. M. Henricks, T. C. Jenkins, and W. C. Bridges. 1992. Serum hormone and metabolite concentrations in fasted young bulls and steers. Domest. Anim. Endocrinol. 9:97-103.

Williamson, D. H., and J. Mellanby. 1974. D-(-)-3-hydroxybutyrate. Pages 1836-1840 in Methods of Enzymatic Analysis. H. U. Bergmeyer, ed. Academic Press, London, UK.

Zinicola, M., M. L. S. Bicalho, T. Santin, E. C. Marques, R. S. Bisinotto, and R. C. Bicalho. 2019. Effects of recombinant bovine interleukin-8 (rbIL-8) treatment on health, metabolism, and lactation performance in Holstein cattle II: Postpartum uterine health, ketosis, and milk production. J. Dairy Sci. 102:10316-10328. https: //doi.org/10.3168/jds.2019-16335. 